\author{
PII S0891-4222(96)00014-5
}

\title{
Long-Term Use of Electrical Aversion Treatment With Self-Injurious Behavior
}

\author{
Pieter C. Duker and Daniel M. Seys \\ University of Nijmegen and Winckelsteegh Residential Facility
}

\begin{abstract}
Twelve severely and profoundly mentally retarded individuals with life-threatening self-injurious behaviors were exposed to electrical aversion treatment using a remotely controlled device. Long-term effectiveness was assessed for periods ranging from 2 to 47 months for the 12 individuals, respectively. The degree of imposed physical restraint was used as the major dependent variable. With two individuals, the treatment failed to suppress self-injurious behavior. With seven individuals, however, suppression was nearly complete in that physical restraints were no longer necessary. With three individuals moderate effects were obtained, in that, although a substantial decrease of imposed physical restraint had been achieved, they still needed daily administrations of electrical aversive stimuli. The results are discusssed in terms of the practical application of this procedure.
\end{abstract}

Self-injurious behavior (SIB) poses a severe threat to one's mental and physical health and creates heavy stress with parents and caretakers. The long-term effects of SIB can hardly be overestimated due to the often (hundreds of) thousands of hits and bangs against the head. Neurological and ophthalmological damages are likely to emerge, not to speak of injuries of surface tissue. The need to treat such behaviors is acknowlegded, although therapists often disagree with respect to the nature of intervention, especially regarding aversive stimuli. Although the debate on the acceptability of aversive stimuli to decrease

The authors gratefully acknowledge the financial support of the Stichting Behandeling en Onderzoek van Zwakzinnigen.

Requests for reprints should be sent to Pieter C. Duker, University of Nijmegen, Psychol. Lab. A6.23, P.O. Box 9104, 6500 HE Nijmegen, The Netherlands. 
SIB and other threatening topographies of behavior continues and ethical arguments tend to dominate the debate, the validity of the argumentation pertaining to the acceptability of electrical aversion therapy (EAT) is undoubtedly related to the long-term effectiveness of the procedure.

Although any stimulus that decreases the occurrence of a response is conceptualized as an aversive stimulus, the present study refers to assessing the effectiveness of EAT, also called electrical shock, with SIB and aggressive behavior.

Since the first published study on EAT by Insalaco and Hamilton (1966), consultation of Database Problem Behavior (Didden, Duker, \& Korzilius, 1993) shows that 46 studies have been published on electrical aversive stimuli to decrease SIB with individuals who are mentally retarded. These studies are all single-case studies. Devices to administer the aversive stimuli differ between therapists, ranging from a cattle prod in the early years to the use of SIBIS (selfinjurious behavior inhibition system [see Linscheid, Iwata, Ricketts, Williams, \& Griffin, 1990] and GED [graduated electronic decelerator; see Israel, von Heyn, Connolly, \& Marsh, 1992]) nowadays.

Long-term data on the use of EAT have appeared lately (von Heyn, Israel, \& Worsham, 1993; Linscheid, Hartel, \& Cooley, 1993; Ricketts, Goza, \& Matese, 1993; Williams, Kirkpatrick-Sanchez, \& Crocker, 1994). Long-term effectiveness of this kind of treatment varies considerably between individuals and devices.

To inform consumers or their representatives fully about this kind of treatment, data collection of long-term effects of EAT on SIB with a larger number of individuals is needed.

Electrical aversion treatment with 12 severely and profoundly mentally retarded individuals who showed high rates of SIB (one individual with aggressive behavior as well) that had resulted in severe physical injuries, such as "cauliflower" ears, severe skin injury, and blindness was studied. Many clients were physically restrained to a such degree that mobility was severely limited. We report on all individuals with whom we conducted EAT since May 1991, yielding follow-up data ranging from 2 to 47 months, for the 12 individuals, respectively.

Data are usually collected within a series of sessions in that the baseline phase can be compared with treatment. Estimation of baseline rates assumes, however, that an individual's physical health is not endangered. However, with 10 of our individuals physical restraints were imposed during the whole day, to prevent them from injuring themselves. One individual, although not physically restrained, had already hit himself to blindness in both eyes before we were consulted. As caregivers' task is, among others, to prevent inflicting physical injury on the individuals whom they care for, the use of physical restraints with this type of individual is most often mandatory. For the above reason, we used the degree of imposed physical restraint as the dependent variable (see Recording), concurrent with traditional data recording. 


\section{METHOD}

\section{Individuals and Setting}

There were 12 individuals ( 6 females and 6 males) involved, C., E., H., W., M., B., R., F., Wi., We., J., and A., with ages ranging from 3 to 43 . All were functioning at a severe or profound level of mental retardation. Nine of them lived in residential facilities throughout The Netherlands. E., We., and J. lived at home. Treatments were conducted at residential facilities, at a community facility, and at home. All individuals had shown SIB since their early years of life. With all individuals, procedures, such as extinction, DRO, water mist, functional movement training, gentle teaching, and numerous sorts of pharmacological agents (e.g., opiate antagonists, antidepressants, and antipsychotics) had failed to reduce SIB. The therapists (i.e., the authors) had been specifically asked by the parents or caregivers for an intervention with EAT, considering this as a last resort.

Consent was obtained from the individuals' relatives and from a human rights committee in all cases. A representative of the state department of health was informed preceding commencement of the treatment and was kept informed during the course of the therapy. Preceding treatment with EAT each individual had undergone a medical check-up.

Functional analysis (Iwata, Dorsey, Slifer, Bauman, \& Richman, 1982) could be conducted with only two individuals (i.e., F. and We.), suggesting multiple functionality of their SIB. Due to the intensity of their behavior, analysis with the other individuals was precluded.

\section{Apparatus}

Shocks were administered remotely with the HSP 3012 (Schoutissen Electronics, The Netherlands). The three parts of the device are: (a) a remote control unit with a battery, which, when the button is pressed, transmits a coded signal to activate the receiver; (b) a receiver $(8 \mathrm{~cm} \times 8 \mathrm{~cm} \times 5 \mathrm{~cm} ; 425 \mathrm{~g})$ consisting of an inductorium, a radio frequency receiver, and a $5.5 \mathrm{~V}$ battery, held in a belt-bag around individual's waist; administration of the electrical stimulus occurs through two circular electrodes (surface size $1 \mathrm{~cm}^{2}$, set $40 \mathrm{~mm}$ apart) attached to individual's upper leg; and (c) a charger, equipped with control lights indicating the condition of the batteries of the remote control unit and the receiver. The apparatus had been approved by the Dutch health authorities.

\section{Recording}

Two types of recording were in effect. First, as an index of the severity of SIB individual's degree of imposed physical restraint was used. Observers were presented with descriptions of physical restraint and asked to indicate the description that would most closely match individual's actual imposed physical 
restraint. Descriptions of physical restraint were presented for the following body parts: head, neck, hands, arms, trunk, waist, and legs/feet. Individual's position could be either ambulant, sitting in a (wheel)chair, or lying in bed. None of the individuals involved stayed in bed during the waking hours. There were 38 different descriptions of the degree of imposed physical restraint. An example of such a description is: “. . . helmet on, while cheeks, ears, eyes, chin, and neck uncovered." Estimates of interobserver reliability yielded a Kappa coefficient of 77 for a group of 50 individuals with SIB and imposed physical restraint. The degree of physical restraint was assessed immediately preceding the initiation of EAT by having two observers independently rate the degree of imposed physical restraint with eight individuals. No physical restraints had been imposed on E., W., F., and We. A second rating was obtained in July 1995, which constituted follow-up lengths ranging from 2 months for A. to 47 months for $\mathrm{C}$.

Second, in conjunction with using the above procedure frequency recording was possible for one $7 \mathrm{~min}$ session with $\mathrm{C}$. while out of restraints and for two 7 min sessions with $\mathrm{E}$., as an estimation of their baseline. W. was already receiving EAT, using the AP-100 Personal Shocker (Farrall Instruments). The aversive consequence of this device was extremely mild and failed to suppress her SIB. No other information was available other than that she would receive several hundreds of mild shocks each day. With F., one 8 min session of frequency recording was used as baseline. Finally, partial interval recording, using 10-s intervals, was used with We. during one session of $26 \mathrm{~min}$. The second assessment was conducted in an identical manner in July 1995.

\section{Procedure}

Treatment. The following phases were in effect. As a first phase, the therapists provided the parents, the staff, and caregivers with information on the rationale of the treatment, showed them videotape(s) on therapists' previous treatments with EAT, and had all people involved feel the electrical stimulus. Following this, the therapists completely or partially removed individual's physical restraint(s) and administered response-contingent electrical stimuli for each occurrence of the target response during one session (i.e., 30-45 min) to assess the response to the electrical stimulus. If initial suppression failed to occur, a second session was scheduled, either the same day, or a couple of days later. During the session(s), the therapist attempted to relax the individual if a panic response or anxiety occurred. Also, the individual was reinforced for escalating intervals of refraining from SIB. Multiple topographies of SIB (e.g., head hitting and head banging) were not treated simultaneously but successively.

As a second phase, given suppression of SIB, the above resulted in introducing the procedure to a case manager, who had a masters degree in psychology. This person was in charge of using the procedure in the individual's natural 
environment (i.e., living group, classroom, home) during a large part or the whole day for a period up to several months. The procedure had the following components: (a) administration of aversive stimuli immediately following the occurrence of the target behavior(s), according to the guidelines given by Carr and Lovaas (1983); (b) differential reinforcement of other behaviors; and (c) supervision and feedback to parents, ward staff, and other professionals on their use of the above components. Also, (d) the case manager conducted oneto-one training sessions with the individual involved as an attempt to establish a repertoire of communicative gestures. It has been demonstrated that establishing a gestural communicative repertoire reduces (moderate) rates of SIB (Duker, Jol, \& Palmen, 1991). Due to shortage of their entry behaviors - individuals had often been restrained for many years - the above training could be initiated with C., M., and F. only. As R. and A. already had some speech, they were excluded from gesture training. Training proved to be effective with $\mathrm{C}$. and F. only.

The final phase refers to monitoring by the two therapists of the use of the treatment during the entire course of time. Regular caregivers and/or parent(s) are now fully in charge of the treatment, while the case manager has been withdrawn. In some facilities the administration of EAT was deliberately limited to a small number of caregivers. Monitoring could vary from phone calls by the authors to repeated on-the-spot observations of the administration of the procedure if there were relapses. The latter resulted in several modifications of treatment during the course of time for those with whom we continued treatment (see Results).

Modifications of the treatment. These had to be undertaken with four individuals. Decisions in this respect were based on monitoring of the suppressive effect of EAT. Two types of modification were in effect, that is, behavioral and pharmacological.

C.: after several months of treatment it was decided that, following five administrations in a row of the electrical stimulus within a time interval of a couple of minutes, she was sent to a time-out room, where she had to stay for 5 min provided that she refrained from SIB. This seemed to maintain low rates of SIB. Also, the addition of a tricyclic antidepressant (i.e., clomipramine) and, later, orgametril, proved necessary, due to a diagnosis of major depression and menstrual complaints, respectively.

E.: a diagnosis of infantile autism, established after commencement of the treatment, prompted us, due to a still modest effect of EAT after 4 months, to administer him an antipsychotic drug. A short withdrawal of EAT indicated the need to continue this treatment.

B.: a relatively large number of aversive stimuli per day continued to be necessary during the course of time (see Results). As we were unsure about her nociception, she was given an opiate antagonist (naltrexone) at $2.0 \mathrm{mg} / \mathrm{kg}$ body weight, which is suppossed, by blocking the production of beta-endorphins (see 
e.g., Thompson, Hackenberg, Cerutti, Baker, \& Axtell, 1994), to lower her pain threshold. A short withdrawal of this drug confirmed its effectiveness on the intensity of her SIB.

R.: after a strong initial decrease of SIB, pneumonia that he contacted seemed to be related to a strong increase of SIB. Due to the emergence of depressive symptoms $\mathrm{R}$. was given a tricyclic antidepressant. The differential effectiveness of this drug on SIB could not be determined.

\section{RESULTS}

Reliability of recording on using the descriptions of imposed physical restraint was calculated by an item-by-item comparison, which revealed a $96 \%$ agreement between the observers for the individuals involved. For behavioral recordings (for C., E., F., and W.) a mean interobserver agreement of $93 \%$ was obtained. We transcribed the descriptions into a pictorial representation of the degree of imposed physical restraint. Figure 1 shows the baseline and the (post)treatment representations of imposed physical restaint and behavioral recordings for each of the individuals.

After 4 and 18 months, treatment was ceased with $H$. and $W$., respectively. Although initial suppression of SIB had been achieved, tolerance for the aversive stimulus seemed to have developed with them. Their degree of imposed physical restraint has, therefore, remained unaltered.

At the other end on the continuum of effectiveness, suppression of SIB was maintained with C., E., F., Wi., We., J., and A. These individuals continued to remain unrestrained and had virtually zero levels of SIB. With E. the shock device could be withdrawn. At the intermediate level of effectiveness, M., although out of restraints for 6 to $8 \mathrm{hr}$ each day, still received 10 to 30 aversive stimuli each day. B., who was maximally restrained to control SIB and aggressive behavior at the initiation of treatment, is still receiving a large number of aversive stimuli per day (i.e., a mean of approximately 50 stimuli per day during the months January through April 1995). These data are, however, somewhat obscured by the fact that the degree of imposed physical restraint with her continues to decrease. Although we initially succeeded in suppressing SIB with R., after several months SIB recurred, while he seemed not to respond to the electrical stimuli anymore. Physical restraint in the form of a cap had now to be imposed, which was necessary to prevent him from inflicting serious injury to his body. Nociception was reestablished during the course of time.

Side-effects. Panic and extreme anxiety (i.e., screaming, crying, attack, escape) initially resulted when the aversive stimuli were given to E., M., Wi., We., and $J$. While relaxing them during a second session, the aversive stimuli succeeded in suppressing the target behavior. A different reaction to the stimulus was presented by F., who froze by refraining from showing any sort of behavior. 


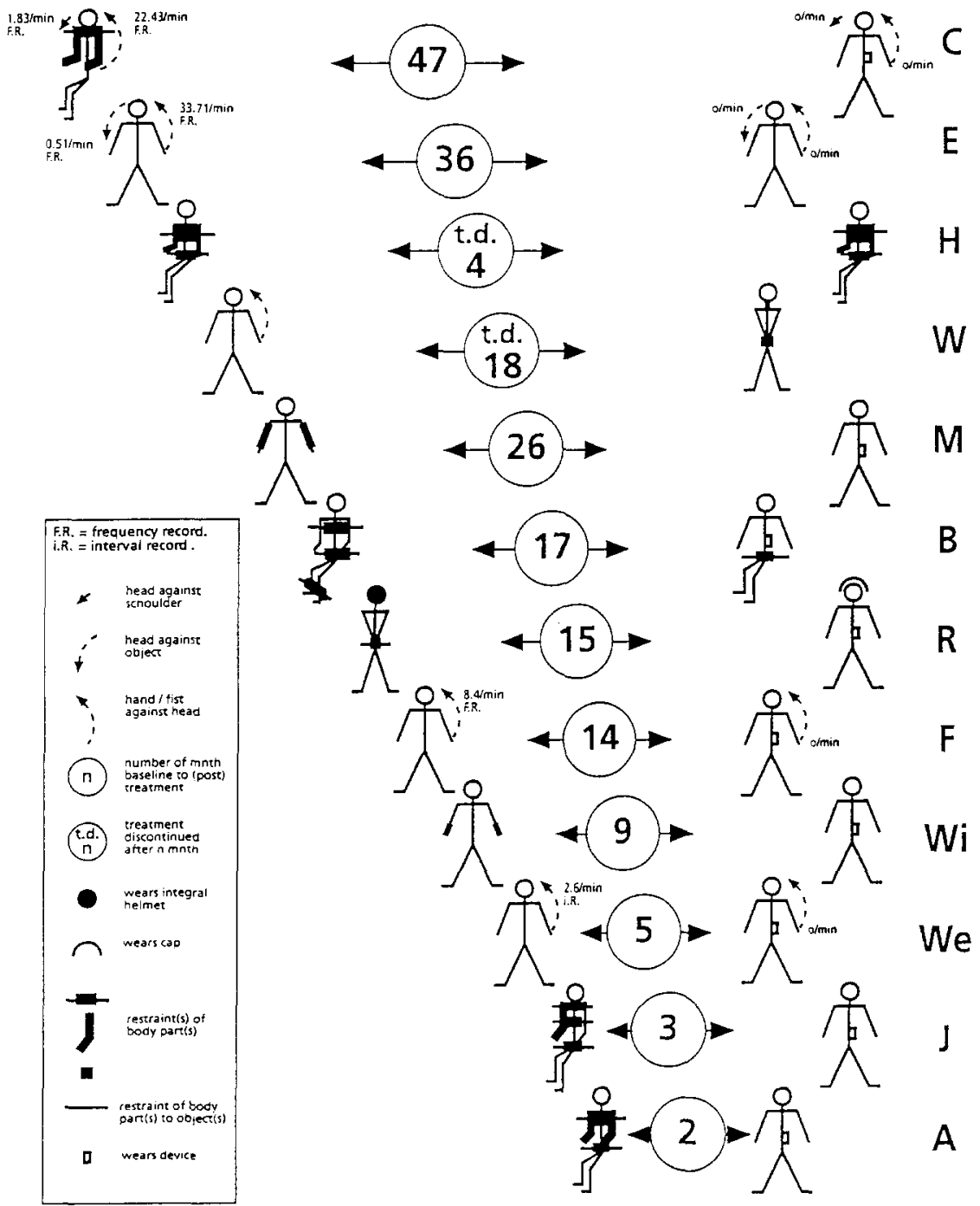

FIGURE 1. Pictorial representation of the degree of imposed physical restraint during baseline and (post) treatment for each of the individuals.

Relaxation and verbal instructions were then necessary. F. successfully underwent surgical treatment of the cataracts at his both eyes. He gained vision in one of his eyes. 


\section{DISCUSSION}

In the present study three categories of effectiveness are distinguished, that is, EAT has been (a) ineffective: this is the case with H. and W.; (b) moderately effective: with M., B., and R., and (c); effective: with C., E., F., We., Wi., J., and $\mathrm{A}$. With the latter four individuals, follow-up lengths are, however, still relatively short.

A general pattern of responding to electrical stimuli can be observed with the present individuals. Initially, individuals tend to show a strong response to EAT by suppressing their SIB. Then, after several weeks a relapse occurs, in that the number of shocks to be given increases. Such an adaptation to the electrical stimulus poses heavy stress on parents, caregivers, and staff, as the individual involved has demonstrated to withhold SIB. Several measures can then be taken, such as administration of opiate blockers (Ricketts et al., 1992), increasing physical restraint, or using antidepressants or antipsychotics, all in conjunction with electrical stimuli. Researchers have also used a stronger electrical aversive stimulus to control such a relapse (e.g., Williams, KirkpatrickSanchez, \& Iwata, 1993). Compelling evidence on the effectiveness of stronger electrical stimuli with severe SIB and aggressive behavior can be found with Israel et al. (1992).

The results of this study are in concert with those obtained by others (e.g., von Heyn et al., 1993; Linscheid et al., 1993). Despite its effectiveness and its apparent simplicity, a treatment with electrical stimuli is not easy to conduct. Difficulties might be encountered in several respects. First, individual's response to the stimulus is often far from unequivocal, in that SIB may even (temporarily) increase. Fear, panic, and even aggressive responses may initially be provoked. Second, during the course of treatment with EAT, the intensity and the form of SIB may alter. For example, the individual may tap his head in a noninjuring manner. This makes it difficult for the therapist to distinguish between behaviors that should be followed by the aversive consequence and behaviors for which aversive consequences should be withheld. Finally, given suppression of SIB, the individual most probably needs to wear the device for a long period of time. With only one individual, that is, E., the device could be withdrawn, whereas the other nine individuals still wear the device (see Figure 1). Most of the individuals seemed highly dependent on the device, in that they would panic or become extremely anxious when the device had to be temporarily removed (e.g., when going into the bath).

It might be argued that any conclusion on the effectiveness of EAT should be based on collecting data within an experimental design. Methodological rigor is often at stake in clinical settings when dealing with life-threatening behaviors. In the present study EAT was initiated at 12 different time points, behavior had often been present for many years, and changes in rate of SIB at the initiation of intervention was most often abrupt; this all may have contributed to the internal validity of the conclusions. Also, traditional recording 
procedures are often inappropriate. Descriptions and pictorial representations of the degree of imposed physical restraint may be an alternative that deserves further attention.

To conclude, EAT may be viable option for individuals who show severe and life-threatening forms of SIB. Further research should focus on how to deal with the adaptation to the electrical stimulus, how to withdraw the device successfully, and how to increase acceptability in the community for the use of EAT for individuals with severe and life-threatening forms of SIB.

\section{REFERENCES}

Carr, E. G., \& Lovaas, O. I. (1983). Contingent electric shock as a treatment for severe behavior problems. In S. Axelrod \& T. Apsche (Eds.). The effects of punishment on human behavior (pp. 221-245). New York: Academic Press.

Didden, D., Duker, P., \& Korzilius, H. (1993). Database Problem Behavior. [Computer software]. Nijmegen: Authors.

Duker, P., Jol, K., \& Palmen, A. (1991). The collateral decrease of self-injurious behavior with teaching communicative gestures to severely/profoundly mentally handicapped individuals. Behavioral Residential Treatment, 6, 183-196.

von Heyn, R. E., Israel, M. L., \& Worsham, R. W. (1993). A comparison of the long-term decelerative effectiveness of two intensities of contingent electric shock on aggressive and health dangerous behavior with individuals with severe behavioral disorders. Unpublished manuscript, Jugde Rotenberg Institute, 240 Laban St., Providence, RI 02909.

Insalaco, C., \& Hamilton., J. (1966). Modification of self-injurious behavior through the use of punishment and reward in a retarded psychotic boy. Unpublished manuscript, Gracewood State School and Hospital.

Israel, M. L., von Heyn, R. E., Connolly, D. A., \& Marsh, D. (1992). A remote-controlled electric shock device for behavior modification. Unpublished manuscript, Jugde Rotenberg Institute, 240 Laban St., Providence, RI 02909.

Iwata, B. A., Dorsey, M. F., Slifer, K. J., Bauman, K. E., \& Richman, G. S. (1982). Toward a functional analysis of self-injury. Analysis and Intervention in Developmental Disabilities, 2, 3-20.

Linscheid, T. R., Iwata, B. A., Ricketts, R. W., Williams, D. E., \& Griffin, J. C. (1990). Clinical evaluation of the self-injurious behavior inhibiting system (SIBIS). Journal of Applied Behavior Analysis, 23, 53-78.

Linscheid, T. R., Hartel, F., \& Cooley, N. (1993). Are aversive procedures durable? A five-year follow-up of three individuals treated with contingent electric shock. Child and Adolescent Mental Health Care, 3, 67-76.

Ricketts, R. W., Goza, A. B., \& Matese, M. (1992). Case study: Effects of naltrexone and SIBIS on self-injury. Behavioral Residential Treatment, 7, 315-326.

Ricketts, R. W., Goza, A. B., \& Matese, M. (1993). A 4-year follow-up of treatment of self-injury. Journal of Behavior Therapy and Experimental Psychiatry, 24, 57-62.

Thompson, T., Hackenberg, T., Cerutti, D., Baker, D., \& Axtell, S. (1994). Opioid antagonist effects on self-injury in adults with mental retardation: Response form and location as determinants of medication effects. American Journal on Mental Retardation, 99, 85-102.

Williams, D. E., Kirkpatrick-Sanchez, S., \& Iwata, B. A. (1993). A comparison of shock intensity in the treamtent of longstanding and severe self-injurious behavior. Research in Developmental Disabilities, 14, 207-220.

Williams, D. E., Kirkpatrick-Sanchez, S., \& Crocker, W. T. (1994). A long-term follow-up of treatment of severe self-injury. Reseanch in Developmental Disabilities, 15, 487-501. 